\title{
Creation of chemically conjugated multichromophoric complexes based on meso- substituted metalloporphyrins
}

\author{
Aleksandr Starukhin ${ }^{1, *}$, Aleksander Gorski ${ }^{2}$, Tatsiana Pavich $^{1}$, Valerii. Kniukshto ${ }^{1}$, and \\ Luiza Gaina $^{3}$
}

${ }^{1}$ B.I. Stepanov Institute of Physics, National Academy of Sciences of Belarus, 220072 Minsk, Belarus ${ }^{2}$ Institute of Physical Chemistry, Polish Academy of Sciences, 01-224 Warsaw, Poland

${ }^{3}$ Babes Bolyai University, 400084 RO, Romania

\begin{abstract}
New multichromophoric complexes based on metallocomplexes of meso-tetraphenylporphyrins chemically conjugated with curcumins and para-terphenyls have been synthesized. Four bands appeared in the luminescence spectrum were assigned. Two first bands in the spectrum at 465 and $497 \mathrm{~nm}$ correspond to the luminescence of curcumin, and the bands at 619 and $677 \mathrm{~nm}$ belong to the luminescence of the meso-substituted metallocomplex of porphyrin. Similar spectral data are manifested in the fluorescence spectra of a complex with four paraterphenyl substituents. For the last one compound the energy transfer between the molecules of the para-terphenyl and metalloporphyrin was postulated with the efficiency of about $20 \%$.
\end{abstract}

The development of new types of organometallic complexes, as well as their chemically conjugated multichrophoric systems is crucial for future applications in various fields of science, technology and medicine. In particular, organometallic complexes are intensively applied as effective photosensitizers in the processes of singlet oxygen generation for using in the photodynamic therapy of cancer [1].

The main idea of our report was to create new promising compounds based on a multichromophoric complexes consisting of a porphyrin macrocycle chemically bonded to a curcumins, known for the high antitumor effect [2] or para-terphenyls. Figure 1 shows the structural formulas of the newly-synthesized metallocomplexes: Zn-5,10,15,20-(tetra-4aminophenyl) porphyrin (Zn-TAPP); Zn-5,10,15,20-(tetra-N-curcumin-4-aminophenyl) porphyrin (Zn-TAPP-(Curc) $\left.)_{4}\right)$ and Zn-5,10,15,20- (tetra-4-N-para-terphenyl-4carboxyphenyl) porphyrin (Zn-TCPP-(ATP $\left.)_{4}\right)$. Fluorescence excitation, fluorescence and phosphorescence spectra of above mentioned compounds have been recorded at ambient and liquid nitrogen temperatures. The data for Zn-TAPP-(Curc) $)_{4}$ is presented on Fig.2. The fluorescence excitation spectra (Fig. 2a) shows that in the spectral range of 400-500 $\mathrm{nm}$ the absorption bands of curcumin are absent.

\footnotetext{
* Corresponding author: starukhin@ mail.ru
} 


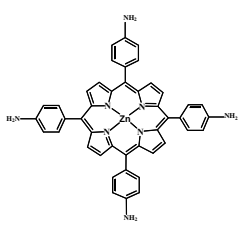

a
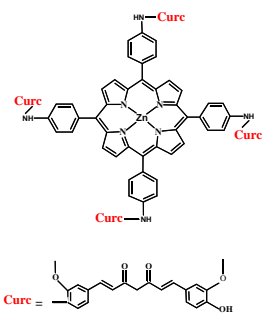

b
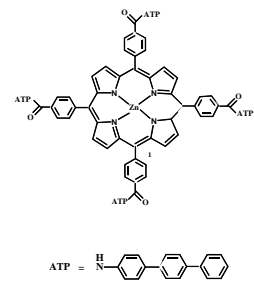

c

Fig. 1. Chemical structures of Zn-TAPP (a), Zn-TAPP-(Curc) $)_{4}$ (b) and Zn-TCPP-(ATP) 4 (c)

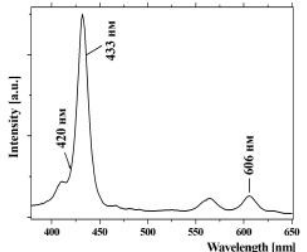

a

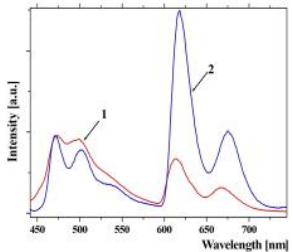

$\mathrm{b}$

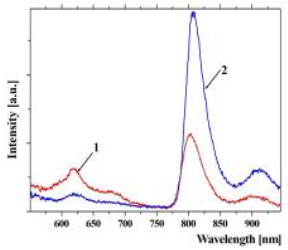

C

Fig.2. Fluorescence excitation at $\lambda_{\text {rec. }}=680 \mathrm{~nm}$ (a), fluorescence: at $\lambda_{\text {exc. }}=420 \mathrm{~nm}-1$; at $\lambda_{\text {exc. }}=433 \mathrm{~nm}$ -2 (b) and phosphorescence: at $\lambda_{\text {exc. }}=420 \mathrm{~nm}-1$; at $\lambda_{\text {exc. }}=433 \mathrm{~nm}-2$ (c) spectra of Zn-TAPP(Curc) 4 in ethanol at $77 \mathrm{~K}$

Four bands are manifested in the fluorescence spectra of Zn-TAPP-(Curc) $)_{4}$ (Figure 2b) in the region from 450 to $700 \mathrm{~nm}$. Two "red" bands at 619 and $677 \mathrm{~nm}$ in the fluorescence spectra of Zn-TAPP-(Curc) 4 correspond to emission of Zn-TAPP (curve 2, fig. 2b). Two "blue" bands in the spectrum with essentially more intensive peaks (at 473 and $503 \mathrm{~nm}$ ) refer to the curcumin emission (curve 1, fig. 2b). Similar dependence is observed in phosphorescence spectra of Zn-TAPP-(Curc) $)_{4}$ (fig. 2c). The band about $620 \mathrm{~nm}$ appears in phosphorescence spectra of Zn-TAPP-(Curc) $)_{4}$ (fig. 2c, curve 1) corresponds to phosphorescence molecules of curcumin. Intensive band about $807 \mathrm{~nm}$ assigned to phosphorescence of $\mathrm{Zn}$-TAPP (fig. 2c, curve 2). The bands in spectrum of $\mathrm{Zn}$-TAPP(Curc) 4 exhibit a more than $5 \mathrm{~nm}$ bathochromic (to long wavelength) shift compared to the complex with one curcumin [3].

New bichromophoric complexes Zn-5,10,15,20-(4-amino-p-terphenylcarboxyphenyl) porphinato (Zn-TACPP-(ATP) 4 ) based on p-terphenylcarboxyphenyl (ATP) conjugated with $\mathrm{Zn}-5,10,15,20$-(tetra-4-carboxyphenyl)porphinato (Zn-TCPP) have been synthesized. Upon creating a complex of Zn-TCPR with ATP, the spectral features of Zn-TCPR are essentially transformed. The effect of the energy transfer between the molecules of the para-terphenyl and metalloporphyrin was postulated with the efficiency of about $20 \%$.

This work was supported by the BRFFI Grant No. F18RA-012.

\section{References}

1. A. jr. Krasnovsky, Biofizika USSR 21, 748 (1976)

2. K. I. Priyadarsini, J. of Photochem. and Photobiol. C: Photochem. Rev. 10, 81 (2009)

3. A. Starukhin, A. Gorski et al. // Web of Conferences 190 Art. No 04023 (2018) 